\title{
A Hidden Markov Model-Based Acoustic Cicada Detector for Crowdsourced Smartphone Biodiversity Monitoring
}

\author{
Davide Zilli \\ Oliver Parson \\ Geoff V Merrett \\ Alex Rogers \\ University of Southampton \\ Southampton, SO17 1BJ, UK
}

\author{
DZ2V07@ECS.SOTON.AC.UK \\ OSP@ECS.SOTON.AC.UK \\ GVM@ECS.SOTON.AC.UK \\ ACR@ECS.SOTON.AC.UK
}

\begin{abstract}
In recent years, the field of computational sustainability has striven to apply artificial intelligence techniques to solve ecological and environmental problems. In ecology, a key issue for the safeguarding of our planet is the monitoring of biodiversity. Automated acoustic recognition of species aims to provide a cost-effective method for biodiversity monitoring. This is particularly appealing for detecting endangered animals with a distinctive call, such as the New Forest cicada. To this end, we pursue a crowdsourcing approach, whereby the millions of visitors to the New Forest, where this insect was historically found, will help to monitor its presence by means of a smartphone app that can detect its mating call. Existing research in the field of acoustic insect detection has typically focused upon the classification of recordings collected from fixed field microphones. Such approaches segment a lengthy audio recording into individual segments of insect activity, which are independently classified using cepstral coefficients extracted from the recording as features. This paper reports on a contrasting approach, whereby we use crowdsourcing to collect recordings via a smartphone app, and present an immediate feedback to the users as to whether an insect has been found. Our classification approach does not remove silent parts of the recording via segmentation, but instead uses the temporal patterns throughout each recording to classify the insects present. We show that our approach can successfully discriminate between the call of the New Forest cicada and similar insects found in the New Forest, and is robust to common types of environment noise. A large scale trial deployment of our smartphone app collected over 6000 reports of insect activity from over 1000 users. Despite the cicada not having been rediscovered in the New Forest, the effectiveness of this approach was confirmed for both the detection algorithm, which successfully identified the same cicada through the app in countries where the same species is still present, and of the crowdsourcing methodology, which collected a vast number of recordings and involved thousands of contributors.
\end{abstract}

\section{Introduction}

The field of computational sustainability, which seeks to apply computer science and artificial intelligence to issues of sustainability, has received great attention in recent years as our planet is under ever stronger environmental, societal and economical pressure (Quinn, Frias-Martinez, \& Subramanian, 2014; Gomes, 2009). Work in this field has striven to bring artificial intelligence research to the real world, implementing practices to promote the sustainability of our environment and to safeguard its living organisms. Towards this goal, the first step is the monitoring of biodiversity, that is the variety of living species in a given environment. Biodiversity is a key measure of the health of an ecosystem, and as land-use and climate change impact on the natural environment, 


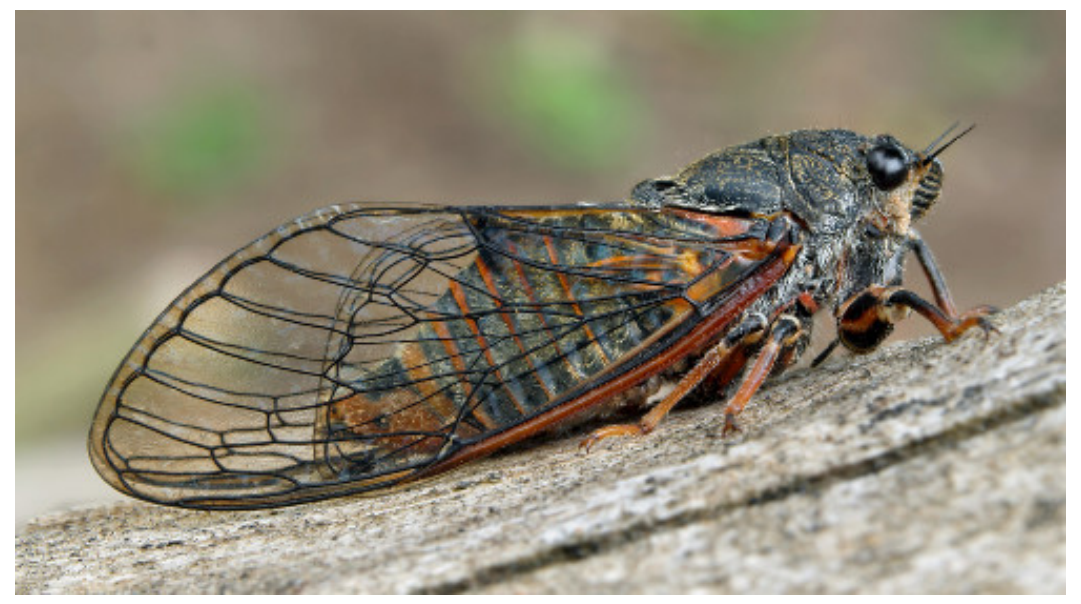

Figure 1: Cicadetta montana. Photograph by Jaroslav Maly, reproduced with permission.

many countries are increasingly seeing the need to monitor and protect it. For example, the UK has formalised this endeavour within the UK Biodiversity Action Plan and has established a priority species list to focus work on a small number of critically important species (Joint Nature Conservation Committee, 2010). One of these, of particular interest in this paper, is the New Forest cicada (Cicadetta montana s. str., see Figure 1), the only native cicada known to the UK, which was first identified in the New Forest, a national park on the south coast of England, in 1812. Despite being well studied at a number of sites in the 1960s, there has been no confirmed observation of the New Forest cicada in the last 20 years (Pinchen \& Ward, 2002). Understanding whether this is simply due to the migration of the cicada to as yet undiscovered sites, or whether the cicada is now extinct in the UK due to climate change or land-use change, is an important question for UK biodiversity research.

Today, traditional approaches for searching for rare species typically require trained ecologists to perform detailed manual surveys. However, the obvious costs of such work have led to significant recent research into automated approaches whereby animals and plants can be classified remotely without requiring that trained experts be in the field. In the case of insects, this is most often performed by deploying fixed sensors with sensitive microphones that record the sounds (or calls) emitted by the insects (MacLeod, 2007). These recordings are then analysed later to automatically identify the insects whose calls were captured. The algorithms for such classification typically range from those that operate solely in the time domain, such as time domain signal coding (Chesmore, 2004; Chesmore \& Ohya, 2004), to those inspired by the literature of human speech recognition (for example Potamitis, Ganchev, \& Fakotakis, 2006; Pinhas, Soroker, Hetzoni, Mizrach, Teicher, \& Goldberger, 2008). The latter typically use a Gaussian mixture model or a hidden Markov model for classification (Leqing \& Zhen, 2010), and perform a number of pre-processing stages, often taken directly from the human speech recognition literature, to extract features from the raw recording. For example, Chaves, Travieso, Camacho, and Alonso (2012) present a state-of-the-art approach that pre-processes the recorded sound to remove un-sounded periods where no insect call is detected, before mapping the raw frequencies to the mel scale, which better represents human hearing. The approach then converts mel scale features back into a pseudo-time domain, called the cepstrum, by calculating a number of mel frequency cepstral coefficients (MFCC), that are then used as features 
for the hidden Markov model (HMM) classification. Such approaches have been shown to classify insects to very high levels of accuracy from clean recordings collected using purpose-built hardware.

The use of such automatic acoustic recognition is particularly appealing in the case of the New Forest cicada, since this insect has a particularly loud high-pitched mating song which, while being close to the upper frequency limit of a normal adult's hearing range and inaudible to most adults over 40 years of age, can easily be detected by conventional microphones. However, the use of fixed sensors to collect these recordings for later analysis is less compelling. The New Forest covers $600 \mathrm{~km}^{2}$, and would require tens of thousands of sensors to exhaustively survey it for potential cicada breeding sites. Furthermore, since the cicada only emits its mating call during the months of June and July, any approach must be able to survey a large area over a short space of time, further decreasing the applicability of fixed sensors.

To address this challenge, we pursue a different approach, which aims to exploit the 13 million day visits to the New Forest that occur each year by the general public to crowdsource the search for the New Forest cicada using a smartphone app. The involvement of the general public in the collection of observations about the natural environment is by no means a recent practice, as records of farmers and clergymen devoted to this activity date back centuries (Miller-Rushing, Primack, \& Bonney, 2012; Brenna, 2011). The start of more structured collection of data, similar to what we know today as the practice of citizen science, can be attributed to the beginning of the 20th century, with events such as the Christmas Bird Count and the foundation of the American Association for Variable Star Observers in 1911 (Silvertown, 2009). However, the Internet has made remote communication and collaboration far easier, and the widespread adoption of smartphones has greatly facilitated the cooperation of amateur scientists around the world to collect and process large amounts of data. In ecology, this method has been a vehicle for such a wide participation of citizens that a plethora of different initiatives has proliferated in the last decade (see for example the survey paper, Dickinson, Zuckerberg, \& Bonter, 2010). An example of this is the iRecord Ladybirds app (Nature Locator, 2013), a system that allows users to collect geo-located photographs of ladybirds and helps them identify the correct species through a series of morphological and taxonomic questions (e.g. colour and number of spots). Records are stored on a database and presented on a public page. However, the app does not attempt to automate the analysis and classification process, outsourcing the task entirely to the users. More recently, the automation of the classification process on a portable device has been attempted for both birds and bats (Jones, Russ, Catto, Walters, Szodoray-Paradi, Szodoray-Paradi, Pandourski, Pandourski, \& Pandourski, 2009). For the former, due to the difficulty in differentiating these calls, work is still in progress and to date no deployed prototype exists. For the latter, a project called BatMobile (Nature Locator, 2012) is starting to implement the automated detection on Apple iOS devices, but the requirement for expensive ultrasonic microphones hinders the accessibility of the tool to the general public on a large scale. The system we propose in this paper is therefore, to the best of our knowledge, the first deployed real-time acoustic species recognition system to run entirely on a mobile device.

However, crowdsourcing acoustic biodiversity using a smartphone app presents a number of challenges. Firstly, smartphones can only be expected to collect short recordings while the user is waiting (30 seconds in our case), in contrast with the always-on recordings collected from fixed sensors. Such fixed sensors would generate much longer recordings (in the order of hours and days) and as a result, existing classification methods are required to automatically remove silent periods from the recording. As a side effect, this can also remove useful time-domain information that can be used to easily differentiate insects with similar frequency calls, especially in lower 
quality recordings from a smartphone. This makes existing methods unsuitable for our purpose. Furthermore, the smartphone app would require that the algorithm provides some real-time feedback to the user as to the identification of the insect being heard. This allows the user to be requested to collect a recording if a cicada is detected, and conversely the user is not required to upload unnecessary recordings if a cicada is not detected ${ }^{1}$. However, low-end mobile devices have limited processing capabilities compared to that of high-end servers, and therefore the previously proposed complex feature extraction methods are not suitably efficient to be run in real-time. In addition, it is essential for an acoustic cicada detector to be able to discriminate between the call of the New Forest cicada and that of other insects commonly found in the New Forest. Two examples of insects with similar calls are the dark bush-cricket, whose call is of a similar pitch to the New Forest cicada but instead chirps with a duration of typically only 0.1 seconds; and the Roesel's bush-cricket, whose call is similar in duration to the New Forest cicada but covers a broader frequency band. Although this scenario involves the detection of relatively few insects compared to existing work, the challenge is to design an approach which can be deployed to the field via low cost hardware for the rediscovery of the New Forest cicada.

Therefore, in this paper we present an algorithm, which we call the Cicada Detection Algorithm (CDA), specifically intended for real-time detection of the New Forest cicada on computationally constrained smartphones. Rather than calculating a number of cepstral coefficients as in existing work, we use the Goertzel algorithm to calculate the magnitude of specific frequency bands, which is an efficient method for approximating individual terms of a discrete Fourier transform (DFT) (Goertzel, 1958). We extract the following three frequency bands: the first centred at $14 \mathrm{kHz}$ corresponding to the strongest frequency component of the calls of the New Forest cicada and the dark bush-cricket, the second centred at $19 \mathrm{kHz}$, where both the dark bush-cricket and the Roesel's bush-cricket are still present, but the cicada is not, and the third centred at $8 \mathrm{kHz}$, which is far from both general background noise (mostly lower than $5 \mathrm{kHz}$ ) and the insects' calls. We then calculate the following two features which form the input to the hidden Markov model: the ratio between $14 \mathrm{kHz}$ and $8 \mathrm{kHz}$ to distinguish between the New Forest cicada and white noise across all frequencies, and the ratio between $19 \mathrm{kHz}$ and $14 \mathrm{kHz}$ to distinguish between the New Forest cicada and the dark bush-cricket. Next, we use a five-state hidden Markov model which explicitly represents the idle period between insect calls, the calls of the New Forest cicada, dark bush-cricket and Roesel's bush-cricket, and also the short pauses during the dark bush-cricket's call. Hence, rather than attempting to independently classify individual segments of insect calls using a complex set of features, we exploit the temporal patterns present throughout the recording using the HMM. We then use the Viterbi algorithm to identify the most likely sequence of insect calls throughout a recording.

We evaluate our approach using 235 recordings of 30 seconds each in duration collected from the New Forest and Slovenia (where the same species of cicada is still present). Unlike standard library recordings, our data set represents the range of crowdsourced data that we are likely to encounter, exhibiting significant noise (e.g. handling noise, road traffic noise, human voice and noise generated by the wind), and insect calls of varying amplitude depending on the proximity of the recording device to the specimen. We show that our approach is able to classify the call of the New Forest cicada in normal environmental conditions with an $F_{1}$ score of 0.82 . Since existing approaches are designed for the batch processing of significantly longer recordings, we compare

1. A 30 s mono recording at 44,100 samples per second, is about $2.7 \mathrm{MB}$; a significant file to upload in areas with poor mobile phone reception where connection rates may be down to $100 \mathrm{kbps}$ or less. 


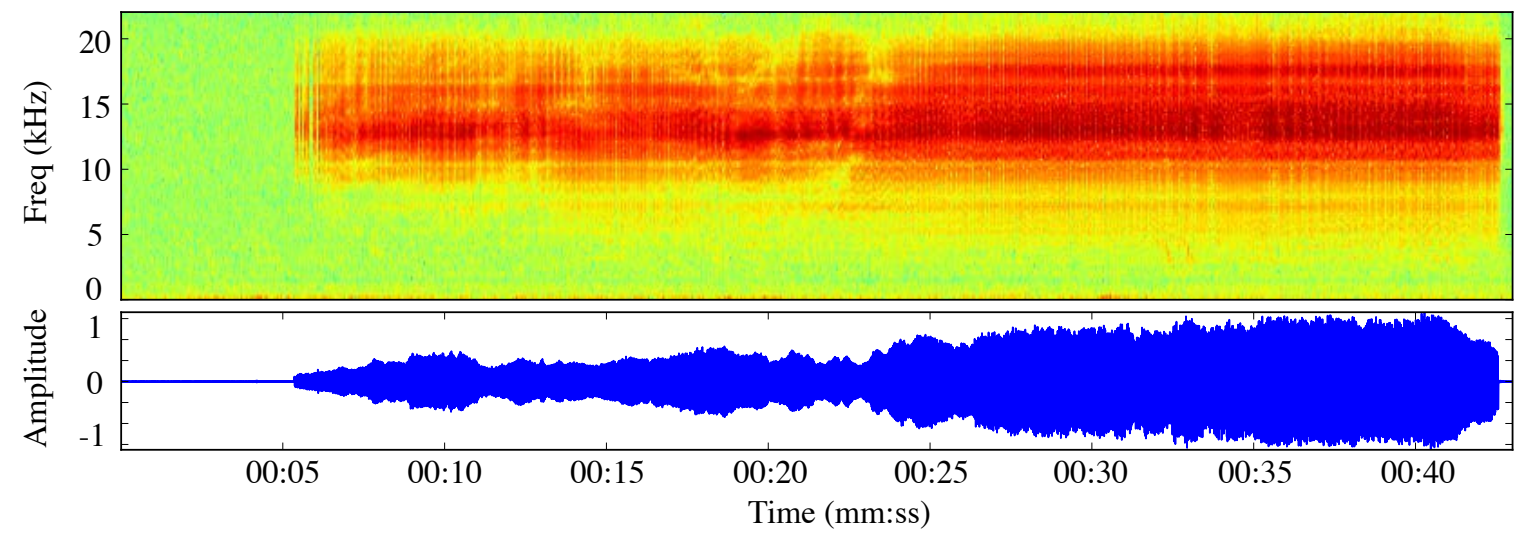

Figure 2: Spectrogram and waveform of a New Forest cicada call (recording by Jim Grant, 1971 and courtesy of the British Library, wildlife sounds collection).

our approach to three variants in order to evaluate the benefit of various components of the pipeline. Our results show that our feature extraction procedure is robust to noise, to the call of the Roesel's bush-cricket and to the call of the dark bush-cricket, and therefore satisfies the requirements of our deployment environment.

This algorithm was implemented on a mobile app, developed for iOS and Android, which was downloaded over 1500 times by members of the public. This culminated in a large-scale trial deployment, with citizen scientists submitting over 6000 reports worldwide. Although the New Forest cicada was not found in this first phase, the accuracy of the detection algorithm and wide geographical coverage achieved via crowdsourcing clearly motivate a second phase of deployment. The same approach can also be applied to the monitoring of many other singing species, and an app that can recognise all British Orthoptera is currently under development.

A preliminary version of our proposed method has also been compared to a state-of-the-art approach for batch classification of insects proposed by Chaves et al. (2012). The comparison, presented by Zilli, Parson, Merrett, and Rogers (2013), shows that our method is considerably more computationally efficient, and therefore better suited for real-time operation. The method proposed in this paper has further improved accuracy and efficiency over that presented in Zilli et al.

The remainder of this paper is organised as follows. In Section 2 we describe our proposed approach, highlighting the different techniques used. In Section 3 we analyse its performance using hundreds of smartphone recordings. In Section 4 we present the first phase of deployment of our approach as a smartphone application, and analyse the coverage of reports collected to date. Finally, we conclude in Section 5 along with our plans for a second phase of deployment to ensure a more complete coverage of the New Forest.

\section{Real-Time Insect Detection Using Hidden Markov Models}

We now give a description of our proposed approach for real-time insect detection. We first describe an efficient method by which individual terms of a DFT can be extracted from raw audio recordings using the Goertzel algorithm. We then describe two features which can be calculated from three individual terms of the DFT to produce a feature vector that can discriminate between the insects of interest and is also robust to environment noise. Next, we formalise the classification of the ex- 
tracted features as an inference problem over a HMM. Last, we propose a five-state HMM designed specifically to capture the temporal patterns of the insects' calls.

\subsection{Feature Extraction Using Goertzel Algorithm}

We observed strong high frequency components in the calls of each of the insects of interest. These frequencies are sufficiently distant from any common background noise, such as wind noise, road traffic or people speaking, to be a reliable indicator for the presence of each insect. Figure 2 shows an example of such a frequency component, in the call of the New Forest cicada which is centred at $14 \mathrm{kHz}$. An efficient approximation of the magnitude of such frequencies can be calculated using the Goertzel algorithm; a method that evaluates individual terms of a DFT, implemented as a second order infinite impulse response filter.

An efficient implementation of the Goertzel algorithm requires two steps. The first step produces a coefficient that can be pre-computed and cached to reduce CPU cycles:

$$
c=2 \cos \left(\frac{2 \pi f}{f_{s}}\right)
$$

where $f$ is the central frequency in question and $f_{s}$ is the sampling rate of the recording.

The second step consists of iteratively updating the values of a temporary sequence $y$ with any incoming sample $s_{n}$ such that:

$$
y_{n}=\operatorname{hamming}\left(s_{n}\right)+\left(c \cdot y_{n-1}\right)-y_{n-2}
$$

where the samples are passed through a Hamming filter, given by:

$$
\operatorname{hamming}\left(s_{n}\right)=0.54-0.46 \cos \left(\frac{2 \pi s_{n}}{N-1}\right)
$$

and the length of the sequence of samples $N$ determines the bandwidth $B$ of the Goertzel filter, such that:

$$
B=4 \frac{f_{s}}{N}
$$

A shorter sequence length $N$ yields a larger bandwidth, at the cost of a noisier output. In practice, we use multiples of 128 samples to match a typical smartphone's audio recording buffer size. For example, a block size of $N=128$ samples gives a bandwidth of just under $1.4 \mathrm{kHz}$. The magnitude $m$ of the frequency band centred at $f$ and with bandwidth $B$ in time slice $t$ is then given by:

$$
m_{t, f}=\sqrt{y_{N}^{2}+y_{N-1}^{2}-c \cdot y_{N} \cdot y_{N-1}}
$$

In terms of computational complexity, this approach shows a considerable benefit compared to the single-bin DFT. An efficient algorithm to compute the latter, the fast Fourier transform, has a complexity of $O(N \log N)$, while the Goertzel algorithm is only of order $O(N)$, where $N$ is the number of samples per window. Moreover, the sample update described in Equation 5 can be processed in real-time, eliminating the need for an independent background thread on the smartphone app and the need to store sample values. 


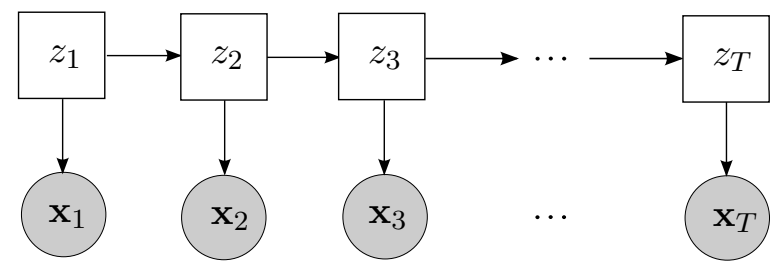

Figure 3: A hidden Markov model. Unshaded square nodes represent hidden discrete variables, while shaded circular nodes represent observed continuous variables. Each $\mathbf{x}_{t}$ is a vector of the two features $x_{t, 1}$ and $x_{t, 2}$.

\subsection{Feature Combination Using Filter Ratio}

The magnitude of the frequency component at $14 \mathrm{kHz}$ is a good indicator of the presence of a New Forest cicada, robust against most background noise, which is normally contained in the lower $5 \mathrm{kHz}$ of the frequency spectrum. However, it may be sensitive to white noise that covers the entire spectrum, such as handling noise. Furthermore, it will not be able to discriminate between the calls of the New Forest cicada and the Roesel's bush-cricket, both of which exhibit a prolonged call at a similar frequency. Therefore, we extract the following three frequencies using the Goertzel algorithm: $m_{t, 8}$ which represents the $8 \mathrm{kHz}$ frequency which is outside the range of both the cicada call and environmental noise, $m_{t, 14}$ which represents the $14 \mathrm{kHz}$ frequency of both the New Forest cicada and the dark bush-cricket, and $m_{t, 19}$ which represents the $19 \mathrm{kHz}$ frequency of only the dark bush-cricket and the Roesel's bush-cricket. We then take ratios of these frequencies to produce two features:

$$
x_{t, 1}=\frac{m_{t, 14}}{m_{t, 8}}, x_{t, 2}=\frac{m_{t, 19}}{m_{t, 14}}
$$

As such, at any point $t, x_{t, 1}$ will be high in the presence of any of the insects considered and tend to one when either no sound is detected in the cicada range or if sound is present across both bands. In addition, $x_{t, 2}$ will be high in the presence of the dark bush-cricket, and tend to zero in the presence of the New Forest cicada. These two features form a $T$-by-2 feature vector which is used for classification by our model. In order to obtain real-time computationally efficient insect identification, we adopt a HMM-based approach to classification as described in the following section.

\subsection{Classification Using Hidden Markov Model}

A HMM consists of a Markov chain of discrete latent variables and a sequence of continuous observed variables, each of which is dependent upon one discrete variable's state (Ghahramani, 2001). Figure 3 shows the graphical structure of a HMM, where the discrete, hidden variables (e.g. idle, cicada singing) are represented by the sequence $z_{1}, \ldots, z_{T}$, and the continuous, observed variables (the features extracted from the audio recording) are represented by the sequence $\mathbf{x}_{1}, \ldots, \mathbf{x}_{T}$. The value of each discrete variable $z_{t}$ corresponds to one of $K$ states, while each continuous variable can take on the value of any real number.

The behaviour of a hidden Markov model is completely defined by the following three parameters. First, the probability of each state of the hidden variable at $t=1$ is represented by the vector 
$\pi$ such that:

$$
\pi_{k}=p\left(z_{1}=k\right)
$$

Second, the transition probabilities from state $i$ at $t-1$ to state $j$ at $t$ are represented by the matrix A such that:

$$
A_{i, j}=p\left(z_{t}=j \mid z_{t-1}=i\right)
$$

Third, the emission probabilities that describe the observed feature, $\mathbf{x}$, given parameters $\phi$, in our case follow a log-normal distribution such that:

$$
x_{t, f} \mid z_{t}, \phi \sim \ln \mathcal{N}\left(\mu_{z_{t}}, \sigma_{z_{t}}^{2}\right)
$$

where $\phi=\left\{\boldsymbol{\mu}, \boldsymbol{\sigma}^{2}\right\}$, and $\mu_{z_{t}}$ and $\sigma_{z_{t}}^{2}$ are the mean and variance of the Gaussian distribution for state $z_{t}$. Figure 4 shows a histogram of data generated by a cicada's song, along with a log-normal distribution fitted to the data. A log-likelihood ratio test on a normal, log-normal and exponential distributions fitted to our data set of cicada songs shows that the log-normal distribution matches the data better than the normal $(F=3512.13, p<0.001)$ and exponential $(F=1516.06, p<0.001)$ distributions. However, despite its long tail, the log-normal distribution still has poor support for data of unusually high magnitude, as are often generated by handling noise. In order to prevent the model from strongly favouring a certain state when a data point is in the extreme of the lognormal distribution, we cap the emission probabilities to capture cases where our data are likely to be poorly represented by this model. The outcome of this is that the likelihood that such data points result from the correct state may be so low that the model triggers a state change even though the transition probability strongly discourages it (by being itself very low). Therefore, we cap the emission probability of such data points such that there is a maximum ratio, initially 100, with which any state can be preferred to another.

Equations 7, 8 and 9 can then be used to calculate the joint likelihood of a hidden Markov model:

$$
p(\mathbf{x}, \mathbf{z} \mid \boldsymbol{\theta})=p\left(z_{1} \mid \boldsymbol{\pi}\right) \prod_{t=2}^{T} p\left(z_{t} \mid z_{t-1}, \mathbf{A}\right) \prod_{t=1}^{T} p\left(\mathbf{x}_{t} \mid z_{t}, \boldsymbol{\phi}\right)
$$

where the model parameters are collectively defined by $\boldsymbol{\theta}=\{\boldsymbol{\pi}, \mathbf{A}, \boldsymbol{\phi}\}$.

We use the Viterbi algorithm (Viterbi, 1967) to infer the most likely sequence of hidden states given the features described. Despite the fact that the number of possible paths grows exponentially with the length of the chain, this algorithm efficiently finds the most probable sequence by maximising Equation 10, with a cost that grows only linearly with the length of the chain.

\subsection{5-State Finite State Model of Insect Call}

We propose a five-state HMM for cicada detection, in which the states consist of: an idle state in which no insect is singing $(I)$, a cicada singing state $(C)$, a state where the dark bush-cricket is chirping $\left(D_{C}\right)$, a short pause in between the dark bush-cricket's chirps $\left(D_{S P}\right)$ and a state in which the Roesel's bush-cricket is calling $(R)$. The emission parameters, i.e. the location $a$ and scale $b$ of the log-normal distribution, are learned empirically using:

$$
a=\ln \left(\frac{\mu^{2}}{\sqrt{\sigma^{2}+\mu^{2}}}\right), b=\sqrt{\ln \left(1+\frac{\sigma^{2}}{\mu^{2}}\right)}
$$




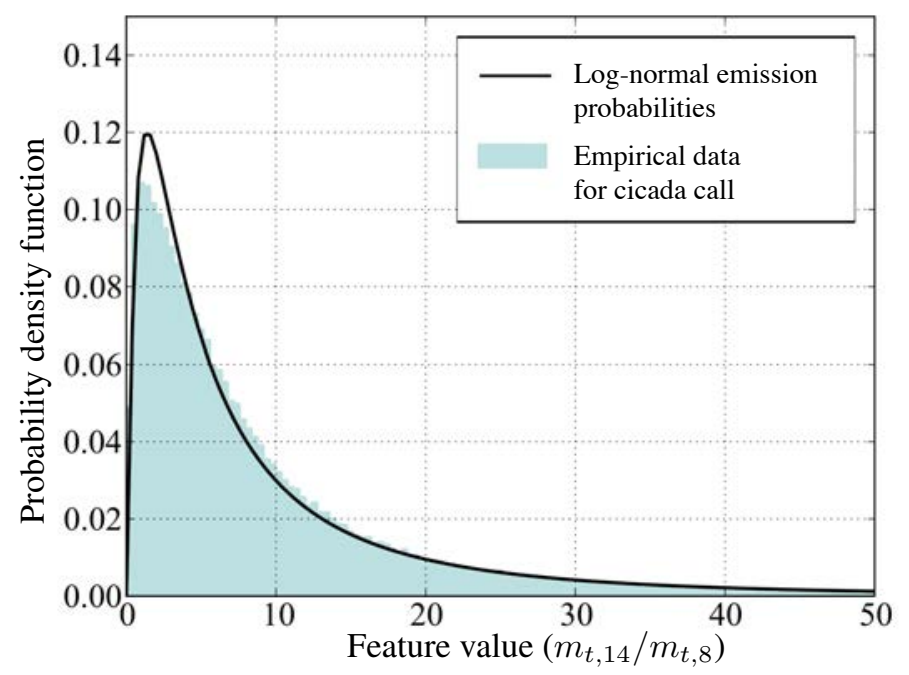

Figure 4: Log-normal distribution of the extracted feature for the cicada call

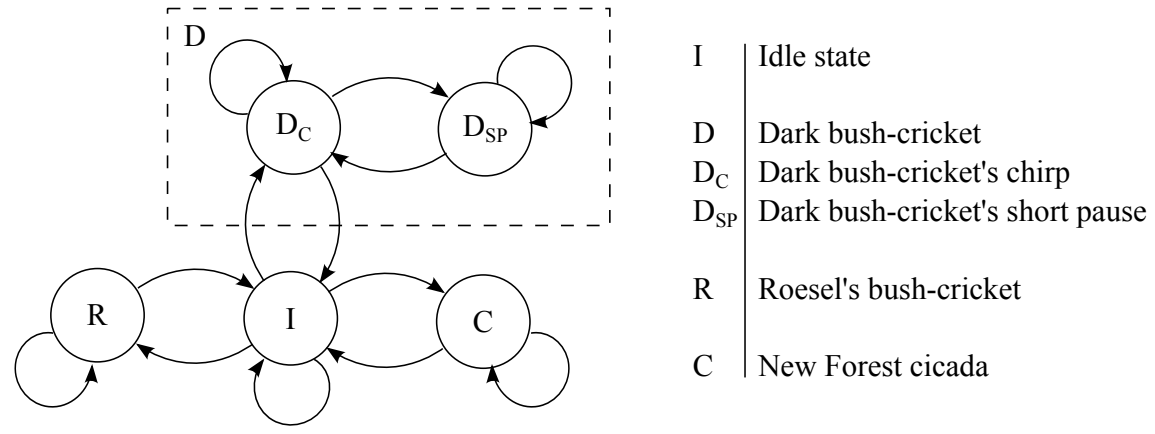

(a) 5-state model used by our approach.

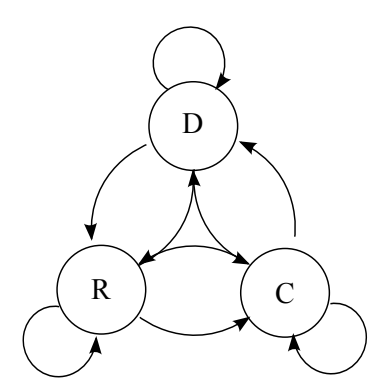

(b) 3-state model.

Figure 5: Comparison of finite state machines.

where $\mu$ represents the mean and $\sigma^{2}$ represents the variance of the data. This manual estimation was originally based on the few recordings the authors had gathered from historical archives, and has therefore been improved with recordings obtained by the deployment of this work, described in the following section.

The transition matrices describing the dynamics of a Markovian process can be represented graphically using finite state machines. Figure 5a shows the five states described above and all possible transitions, where those with non-zero probability are represented by arrows connecting two states. Our model explicitly represents the silence between the dark bush-cricket's chirps, which is essential information for distinguishing between the calls of the New Forest cicada and dark bushcricket. This is in contrast to existing batch classification methods which remove such silent periods of a recording in order to improve the computational cost of the operation and classify only sounded periods of the sample file (Chaves et al., 2012). These methods also employ a feature extraction process whereby they compute a number of mel-frequency cepstral coefficients for each species in the model, making the process scalable to several insects, at the cost of higher computational complex- 
ity. In contrast, the method we proposed in Section 2.1 is more closely tailored to the requirements of our scenario, producing the improvement in efficiency necessary for our mobile application. Figure $5 \mathrm{~b}$ shows a variant of our approach where the silent states have been removed, against which we compare our approach in the following section. Furthermore, the HMM could be arranged so as to be fully-connected, allowing transitions between states that are otherwise disconnected (for example between a Roesel's Bush-cricket and a Dark bush-cricket). However, this confuses the model between states that have very similar emission probabilities, without providing any improvement in accuracy. We therefore exclude this variation from the comparison in the following section.

\section{Empirical Evaluation Using Smartphone Recordings}

We introduce three variants of the approach described thus far that, following the practices in the literature, motivate the choices made to construct our Cicada Detection Algorithm. The first variant uses the same approach as proposed in Section 2, but the three raw frequencies, as opposed to their ratio, are used directly as features (CDA raw frequencies). The second variant removes un-sounded periods from the recording and, as such, segments it into individual calls. It then applies the 3-state model shown in Figure $5 \mathrm{~b}$ to classify the insects (CDA silence removed). The third approach does not apply a HMM at all, and instead uses the ratio of frequencies to directly identify the most likely state, given only the instantaneous emission probabilities of the features. As such, this method can be considered as a mixture model, since each time slice is classified independently. This method is considerably more computationally efficient, at the cost of losing the information of the time domain.

We evaluate the accuracy of each approach using a collection of 235 recordings taken by citizen scientists using smartphones from the New Forest (the only known UK habitat of the New Forest cicada) and by the authors of this paper in Slovenia (where the same species of cicada is still present) over the summer of 2013. Each recording is 30 seconds long, and in most cases contains a call of either the New Forest cicada, a dark bush-cricket or a Roesel's bush-cricket. Some recordings contain different types of noise, including people speaking, walking, calls of birds, handling noise and even people mimicking the sound of the cicada. In contrast to existing recording libraries, this data set represents the typical quality of crowdsourced data, exhibiting significant noise and insect calls of varying amplitude depending on the proximity of the recording device to the specimen. Each recording was later labelled by domain experts as containing either one or none of the insects of interest. Although multiple insects in the recordings will not make the classification fail, we consider only one singing insect per recording. If more than one is present, we set the ground truth across the 30 -second recording as the longest or loudest singing insect, therefore taking the state active for the longest period as the outcome of the model. Since the emission probabilities in our model are purposely tuned, we do not require any training data as such, and hence we use the entire data set as test data. We describe the deployment of the smartphone app used to collect this data in more detail in Section 4.

We assess the accuracy by which each approach can correctly classify the cicada using the standard precision, recall and $F_{1}$ score metrics. The precision represents the fraction of recordings in which the approach detected the cicada as singing when it was in fact singing, while the recall represents the fraction of recordings in which the cicada was singing that were correctly detected. 


\begin{tabular}{cccc}
\hline Approach & Precision & Recall & $F_{1}$-score \\
\hline CDA & 0.66 & 0.78 & 0.82 \\
CDA raw frequencies & 0.46 & 0.94 & 0.62 \\
CDA silence removed & 0.62 & 0.99 & 0.75 \\
Mixture model & 0.61 & 0.65 & 0.67 \\
\hline
\end{tabular}

Table 1: Accuracy metrics of cicada detection

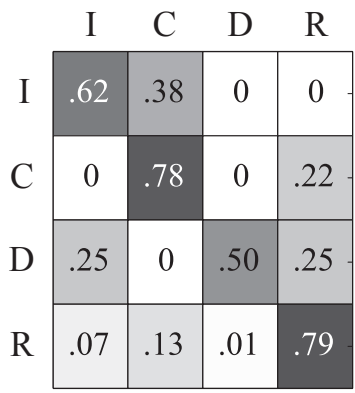

(a) CDA

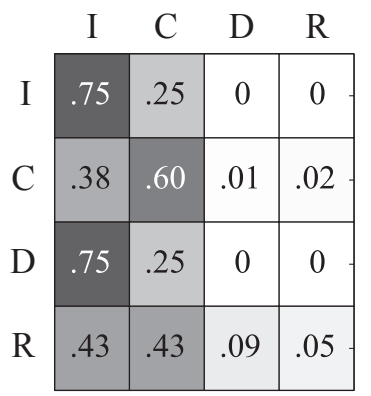

(b) CDA raw frequencies

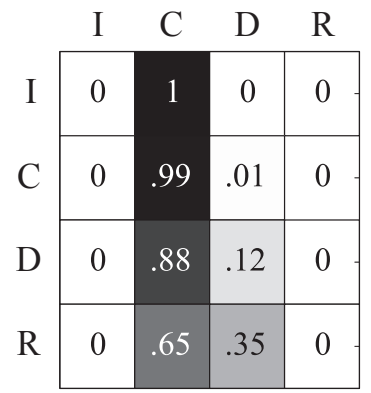

(c) CDA silence removed

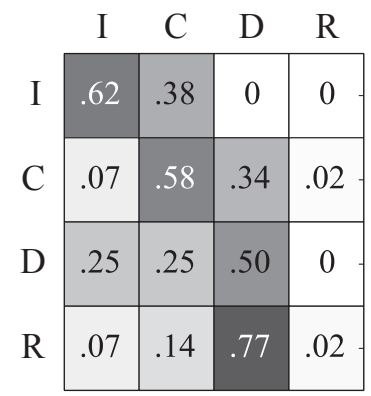

(d) Mixture model

Figure 6: Confusion matrices for the four variants of the detection algorithm. On the y-axis, the actual class; on the $\mathrm{x}$-axis, the predicted class.

Precision and recall are defined as:

$$
\text { precision }=\frac{t p}{t p+f p}, \quad \text { recall }=\frac{t p}{t p+f n}
$$

where $t p$ represents the number of correct cicada song detections, $f p$ represents the number of cicada song detections when it was actually not singing, and $f n$ represents the number of cicada songs which were not detected. This work is primarily concerned with the detection of the New Forest cicada, and as such other insects are modelled in order to avoid false positive detections of the New Forest cicada. We also use the $F_{1}$ score, which represents a weighted combination of precision and recall, defined as:

$$
F_{1}=2 \cdot \frac{\text { precision } \cdot \text { recall }}{\text { precision }+ \text { recall }}
$$

Table 1 shows the precision, recall and $F_{1}$ score metrics of our approach compared to the three variants over the data set of recordings from the New Forest and Slovenia. Similarly, Figure 6 reports the true and false positives, with real values along the $\mathrm{y}$ axis and predicted class along the $\mathrm{x}$ axis. It can be seen that our approach (CDA) achieves an $F_{1}$ score of 0.82 , and as such outperforms each benchmark variant, visually apparent from the darkness along the main diagonal in Figure 6a. In contrast, the variant of our approach which uses the raw frequency measurements as the HMM feature vector (CDA raw frequencies) receives an $F_{1}$ score of 0.62 . This is a result of the approach's lack of robustness to noise, such as handling noise, as shown by the high number of false positives in Figure 6b. Furthermore, the variant of our approach which removes the silent periods (CDA silence removed) receives an $F_{1}$ score of 0.75 . Although this appears as positive result, Figure $6 \mathrm{c}$ highlights 
its lack of ability to discriminate between the dark bush-cricket and the New Forest cicada. This method, as well as the raw frequencies approach, favour the New Forest cicada, scoring a good true positive rate but consequently also a high false postive rate. Finally, the mixture model method receives an $F_{1}$ score of 0.67 because the lack of transition probabilities leaves the decision to the emission probabilities only, not utilising the information contained in the time domain, making the number of true and false positives more equally distributed (Figure 6d). Insects with similar emission probabilities, such as the Roesel's bush-cricket and the dark bush-cricket, will therefore be difficult to classify with this method. It should be noted however that this approach is considerably more computationally efficient, as it decides on the most likely state instantaneously and without traversing the entire recording.

Figures 7, 8, 9 and 10 show a comparison of the four approaches over a sample recording for each of the four species in the recordings analysed. The top plot of each figure shows a spectrogram with the time domain on the $x$-axis, and the frequency domain on the $y$-axis, with the magnitude of the frequency bins varying with the colour of the plot. Subsequently, the figure shows the most likely state identified by each approach. In each plot, the states are labelled as in Figure 5a, where $I$ represents the un-sounded idle state (if present), $C$ represents the cicada's song, $R$ represents the Roesel's bush-cricket and $D_{C}$ and $D_{S P}$ the dark bush-cricket's chirping and short pause states, respectively. The gaps in the silence-removed variant correspond to unsounded periods.

Figure 7 shows that classifying the cicada is easier for the HMM-based methods, as the call lasts for a long period without interruption and is clearly distinct from background noise. A more noisy recording would cause the raw-frequency approach to fail. The mixture model approach struggles to distinguish between the cicada and the dark bush-cricket call, since they are similar in features but different in the time domain, which this model does not capture. Figure 8 shows how the variants are more sensitive to noise than the CDA for different reasons. The raw frequencies approach doesn't filter out background noise, while the mixture model triggers a cicada state even for a very short noise in the right frequency band. The silence-removed method is only active in the short period of higher background noise, and not having an idle state, it is forced to classify the sound as any of the sounded states. Figure 9 shows how, when silence is removed, a Roesel's bush-cricket becomes very similar to a dark bush-cricket, having very similar emission probabilities. The same perception is observed by the mixture model, that doesn't have a perception of time. Similarly, Figure 10 shows that the dark bush-cricket is difficult to classify for the mixture model and the approach with silence removed, as explained thus far. Moreover, it shows how a trade-off between a very quiet insect (visible throughout the recording) and no insect must be made, as the insect could be at any distance from the microphone, and thus there is no limit to how quiet it may be.

The analysis of each of the 235 recordings is presented in detail on the project's web page, together with the parameters of the HMM, the audio file, and information about the recording device ${ }^{2}$.

\section{Automated Classification on a Smartphone App}

We deployed the insect detection algorithm within a smartphone app that enables wide participation in the search for this critically endangered species. This process, often referred to as citizen science, attempts to leverage the widespread presence of users willing to participate such that they act as a distributed network of sensors, while learning about the scientific process behind a certain research,

2. Result at http://www. newforestcicada.info/devdash. The data can be used free of charge, provided that the New Forest Cicada Project is attributed according to the Creative Commons Attribution (BY) licence. 


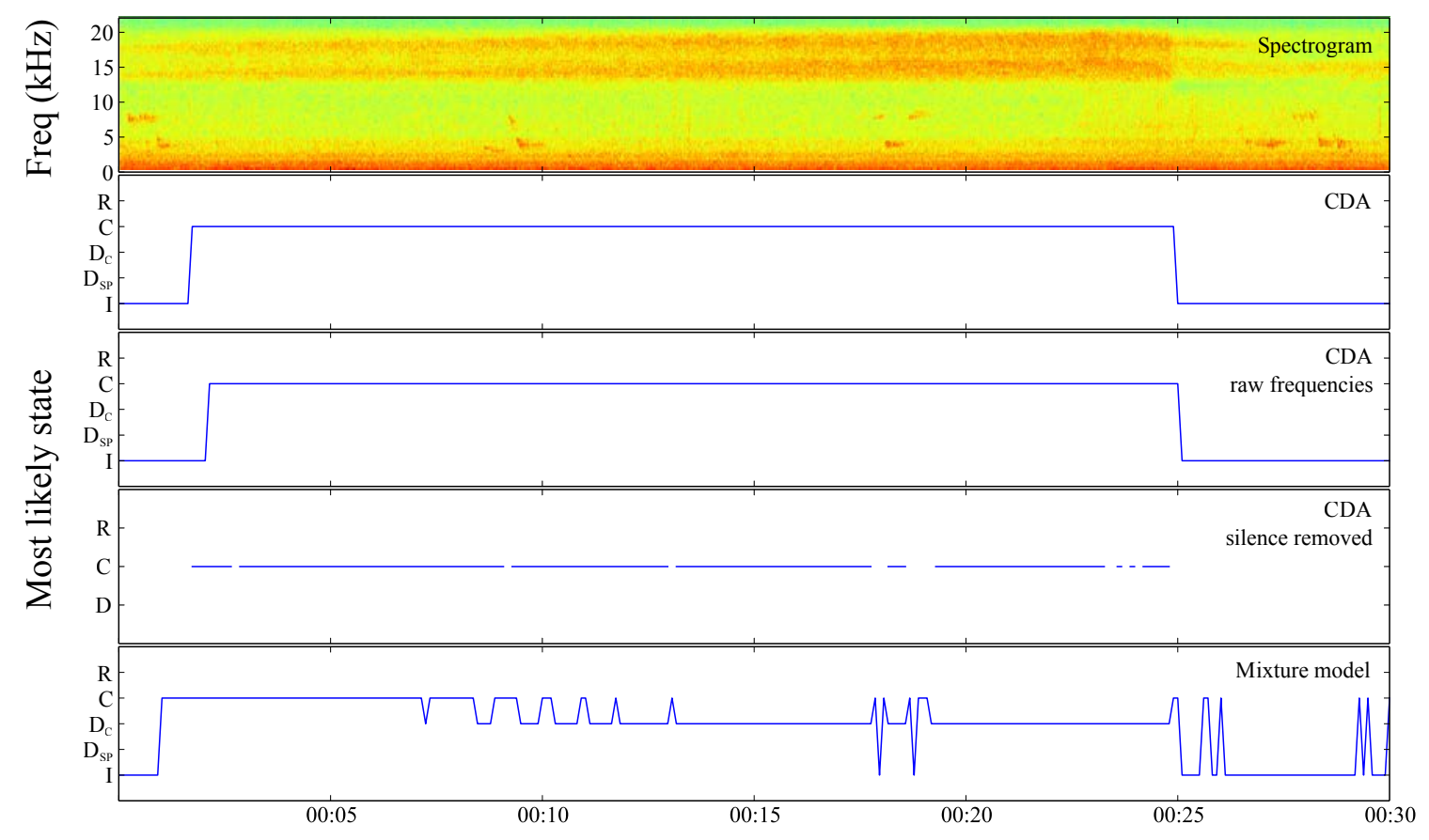

Figure 7: Model comparison on a New Forest cicada recording

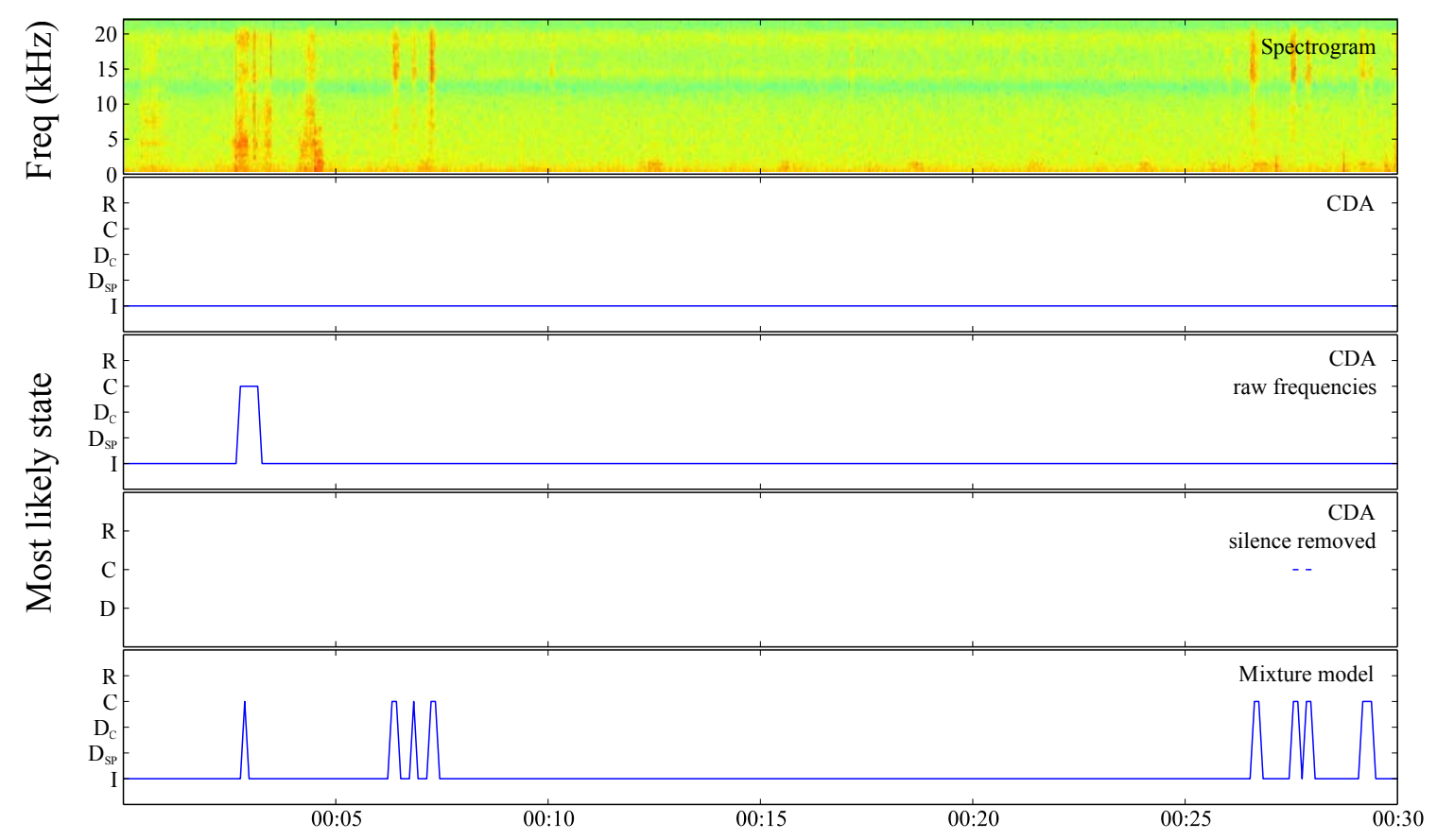

Figure 8: Model comparison on a recording with no singing insect 


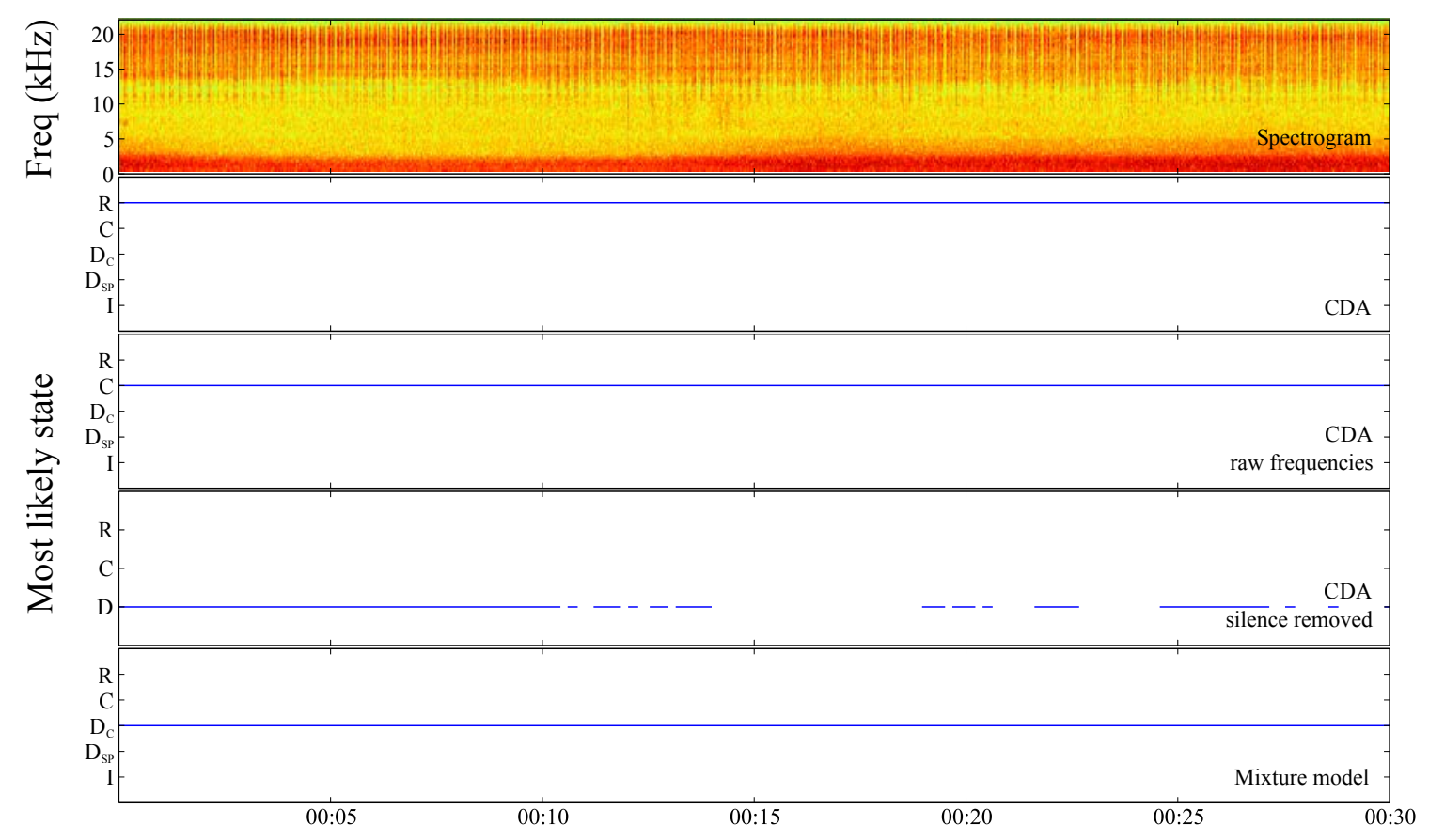

Figure 9: Model comparison on a Roesel's bush-cricket recording

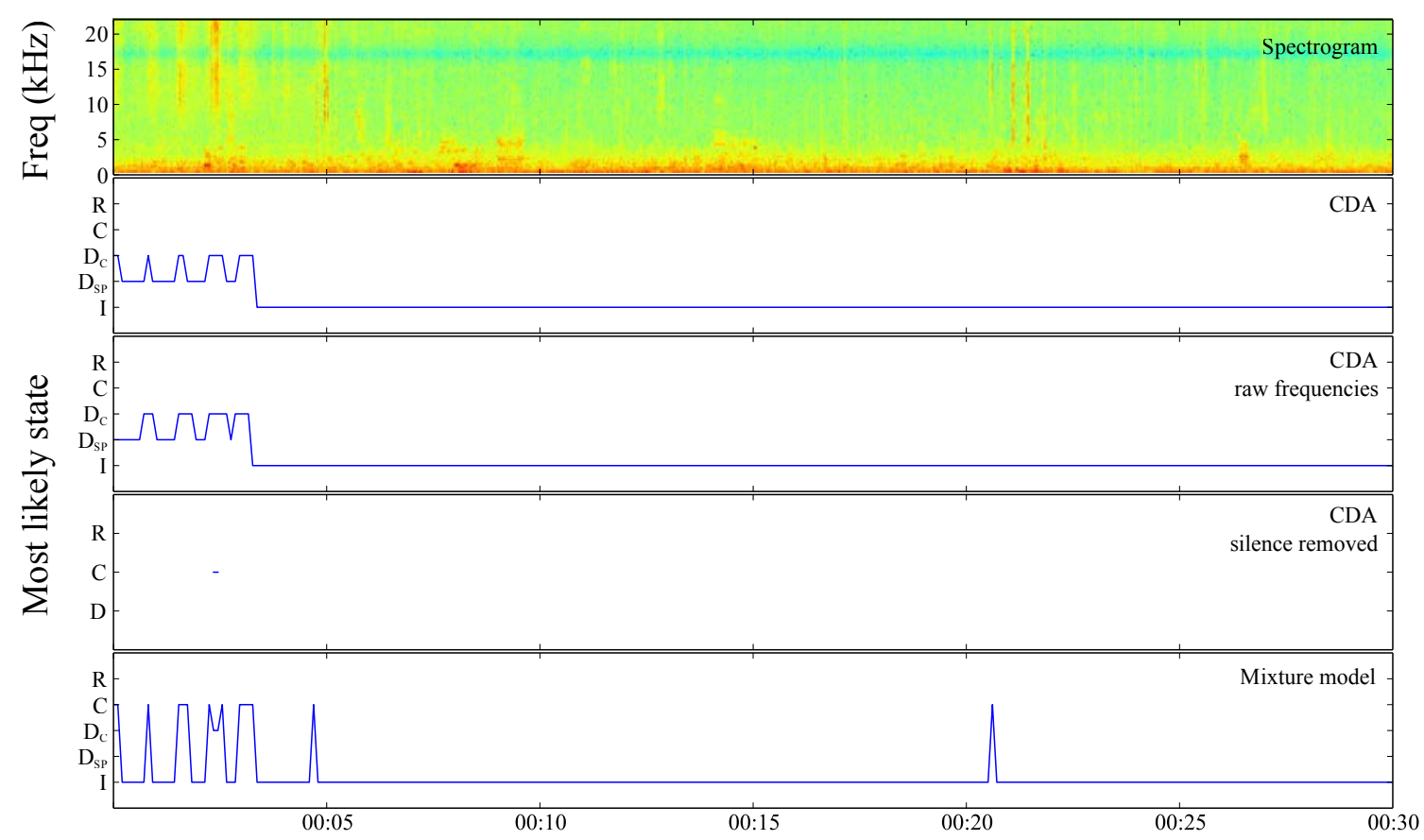

Figure 10: Model comparison on a dark bush-cricket recording 


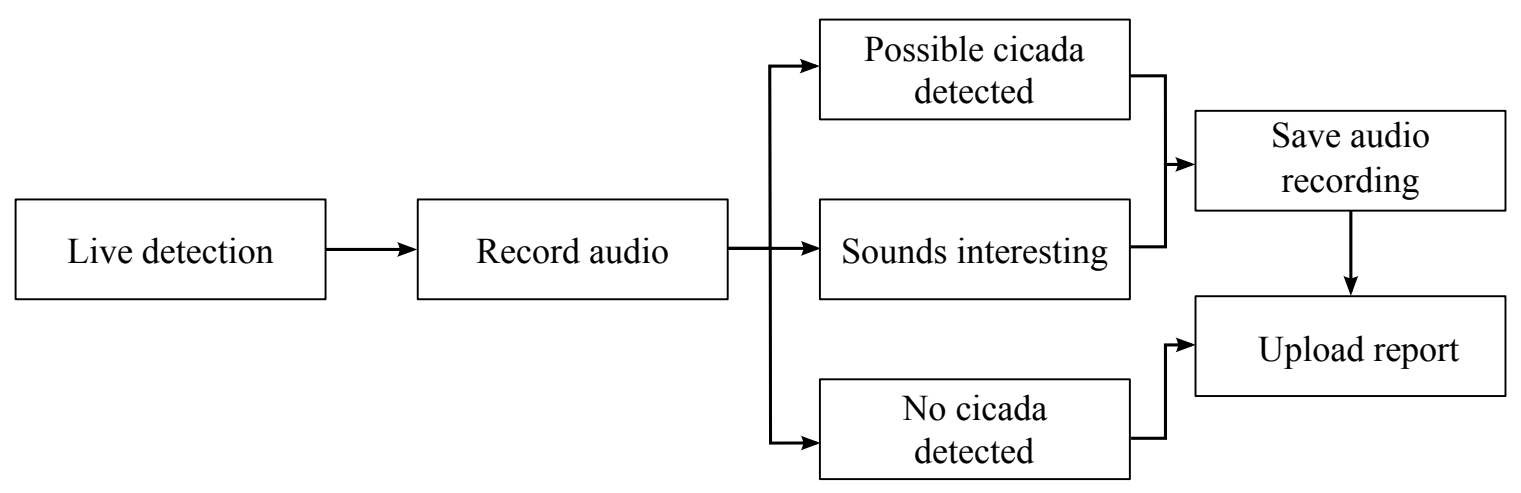

Figure 11: Flow of the detection and classification process on the app.

in this case the automated identification of species for biodiversity monitoring. Examples of the communities to which this app caters include tourists and visitors to the New Forest National Park, local residents and bug enthusiasts. In order to maximise the number of citizen scientists taking part, it was essential for the app to be compatible with a wide range of hardware, in addition to being simple and unobtrusive to use. Therefore, we released both an iPhone and Android client, which ensures compatibility with over $80 \%$ of smartphone users (Go-Gulf, 2012). Furthermore, we designed the app to be simple to use, require user consent when recording audio and to constrain usage of both battery and mobile data usage.

Figure 11 shows an overview of the flow of interaction when a user takes a recording with the app. When a user first opens the app they are presented with the live detection screen, which displays a graphical representation of the audio signal entering the microphone, in the form of a circular spectrogram, and an immediate feedback on the presence of a singing cicada, obtained through the output of the mixture-model described in Section 2. Upon selecting to start an audio recording, the user is shown the current progress through the 30 second recording. On completing the recording, the CDA is run and the user is presented with one of three possibilities: a possible cicada detected screen, a sounds interesting screen (which notes that the algorithm has detected an insect that is not the cicada), or the no cicada detected screen (where nothing known was found). A report of the survey is saved locally and uploaded upon connecting to the Internet. If the recording contains any of the known insects, the user is asked for their consent to upload the recorded audio.

\subsection{Stages of Real-Time Classification}

In order to capture a sound to be fed to the automated classifier, the user is presented with an intuitive interface, summarised in Figures 12 and 13 and detailed as follows:

\subsubsection{Live Detector}

Figure 12a shows the detector screen, which appears upon loading the app. A crucial difficulty for a human to detect the New Forest cicada's call is the fact that the pitch is too high for most people to hear, since the central frequency is at the limit of the hearing range of the average 40 year old. To address this issue, this tab presents a visualisation of the sound drawn as a circular spectrogram. In the centre, the cicada logo lights up when a call is detected, triggered by the instantaneous output of the mixture model described in Section 2, updated every 128 samples from the microphone. Twenty 


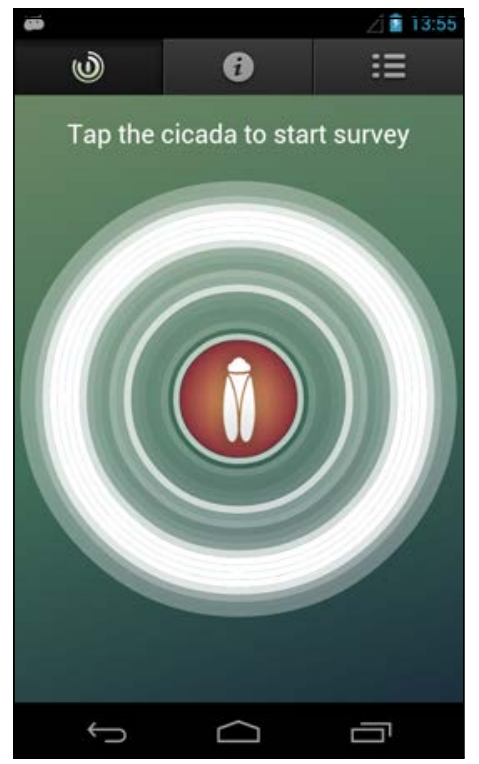

(a) Live detector

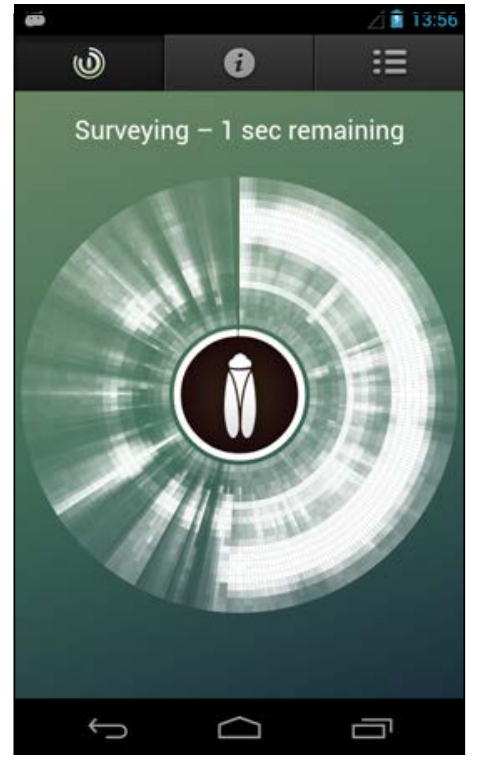

(b) Audio recording

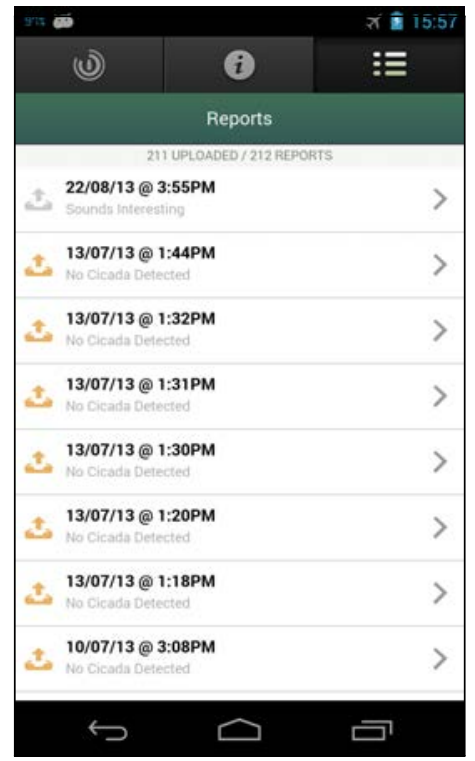

(c) Upload recording

Figure 12: Three screens of Cicada Hunt on Android. On the left, a cicada singing lights up the icon and the frequency bands around $14 \mathrm{kHz}$. In the middle, a cicada was singing during a survey and stopped shortly after 15 seconds. On the right, the latest survey is waiting to be uploaded.

concentric circles around it represent twenty frequency bands of the spectrum, centred from 1 to $20 \mathrm{kHz}$ with a bandpass of $1.4 \mathrm{kHz}$, extracted with 20 Goertzel filters, which ensure rapid updating of the interface. Each of these becomes brighter with a higher signal strength (i.e. a louder sound at that pitch) and paler when the band is quieter. The outer bands, roughly from 12 to $18 \mathrm{kHz}$, are those triggered by the cicada call, producing the distinctive effect shown in Figure 12a. Tapping the cicada icon at the centre of the app starts a 30-second survey, during which the sound is recorded and then analysed by the algorithm described in Section 2. This idea is core to the interface, as it encourages users to stop and wait in silence, thus maximising the chance of detecting the required sound. The choice of 30 seconds strikes a balance between the length of the cicada call and the amount of time a user-mostly an occasional visitor to the forest—can be expected to stand still and in silence.

\subsubsection{Audio RECORDING}

Figure $12 \mathrm{~b}$ shows the screen shown during a 30 second audio recording. Once the recording has finished, the audio is analysed by the HMM-based algorithm described in Section 2. Depending on the result of the classification, the user is shown either the no cicada detected screen, the sounds interesting screen or the possible cicada detected screen.

\subsubsection{UPLOAD RECORDING}

Figure 12c shows a list of reports which have been saved locally. Each report is geo-tagged and time-stamped, and saves a unique identifier of the phone as well as basic information about the device. The report also saves an uncompressed $44.1 \mathrm{kHz} 16$ bit PCM WAV sound recording in the 


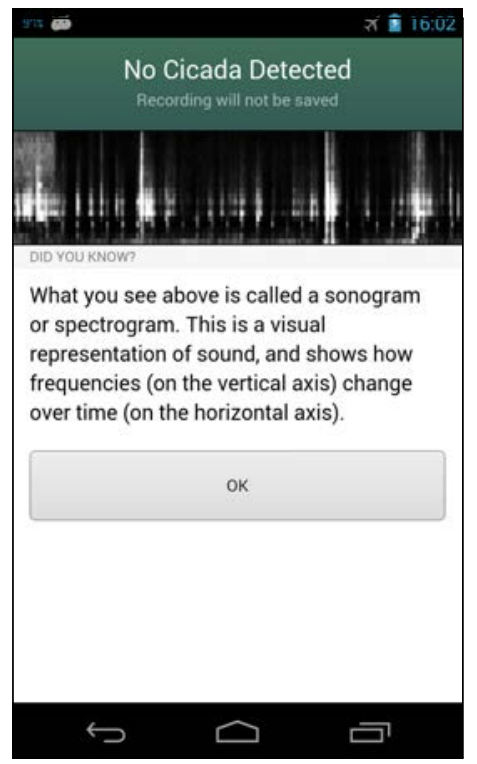

(a) No cicada detected

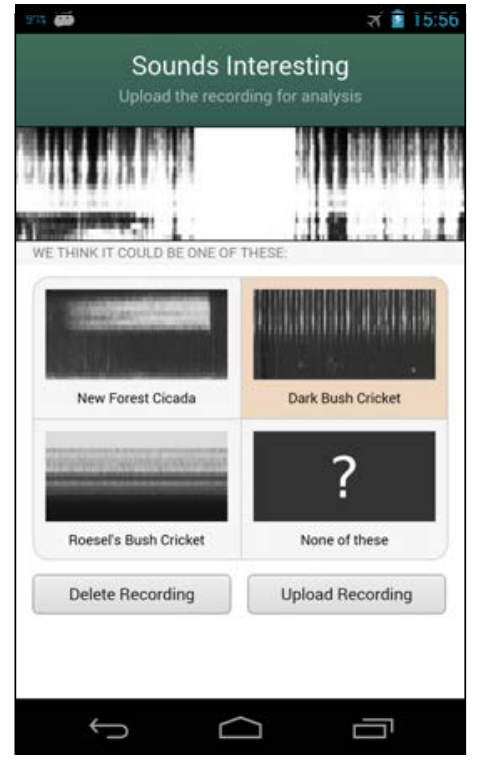

(b) Sounds interesting

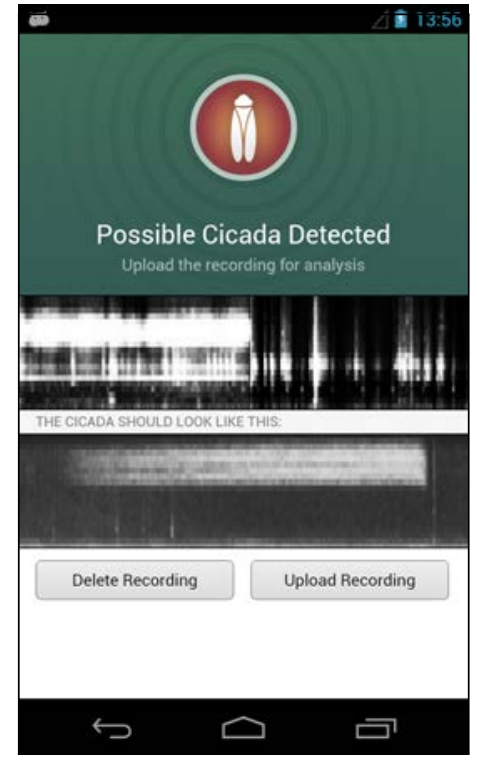

(c) Possible cicada detected

Figure 13: Three possible outcomes of the CDA, where no known insect is found, an insect that is not a cicada is found, or the cicada itself is found.

case that either the cicada or another insect is found, provided the user has granted permission to do so. Since the audio recording requires $2.7 \mathrm{MB}$ on disk, it is deleted from the smartphone as soon as the report is sent to the server to minimise the storage space required on each smartphone. Last, a low-resolution spectrogram is saved in all cases, constructed from the combination of the output of 20 Goertzel filters over the 30 seconds survey, saved every 128 samples. This constitutes the easiest way for a human to check for the presence of the cicada and avoids any privacy concerns (speech could not be reconstructed from such a spectrogram). Moreover, the payload of the image file, saved in Base64 (Josefsson, 2006), is around $15 \mathrm{~KB}$ and therefore much lighter than a raw sound recording. Once an Internet connection becomes available, the report is uploaded to the project's servers, where it is available to the research team to analyse further.

\subsubsection{No Cicada Detected}

Figure 13a shows the screen shown if nothing was detected. A fact about the cicada, its habitat, the New Forest or the technology behind the app is shown to provide an informative notion, encouraging the user to try again. This intends to both support the morale of the user who is receiving negative results, and to provide educational content so that the citizen scientist receives some information in exchange for the work they have performed.

\subsubsection{SOUNDS INTERESTING}

Figure 13b shows the screen displayed when another insect is detected, whose call is similar to that of the New Forest cicada. At present, the app encompasses two other insects present in the New Forest: the dark bush-cricket and the Roesel's bush-cricket. The user is shown a spectrogram of a typical call of these insects, as well as a spectrogram of what they have just recorded, and they are 


\begin{tabular}{lccccc}
\hline Device & OS & Filtered & Silence (SEM) & Cicada (SEM) & Ratio (SEM) \\
\hline iPhone 4 & iOS & No & $1.623(0.075)$ & $13.047(0.327)$ & $8.041(0.442)$ \\
iPhone 5 & iOS & No & $1.897(0.076)$ & $14.793(0.388)$ & $7.800(0.373)$ \\
iPhone 4S & iOS & No & $1.466(0.050)$ & $10.549(0.337)$ & $7.196(0.336)$ \\
iPhone 3 & iOS & No & $1.469(0.047)$ & $10.539(0.430)$ & $7.173(0.372)$ \\
HTC Desire & Android & No & $0.844(0.041)$ & $4.255(0.265)$ & $5.041(0.397)$ \\
Xperia Mini & Android & No & $2.480(0.155)$ & $10.190(0.262)$ & $4.109(0.277)$ \\
Moto A953 & Android & No & $2.015(0.104)$ & $5.845(0.148)$ & $2.901(0.167)$ \\
Galaxy S3 & Android & No & $1.374(0.038)$ & $3.279(0.088)$ & $2.387(0.093)$ \\
Xperia Z & Android & No & $0.951(0.032)$ & $1.971(0.059)$ & $2.072(0.094)$ \\
HTC One S & Android & No & $1.466(0.040)$ & $2.915(0.085)$ & $1.988(0.079)$ \\
Nexus 4 & Android & No & $0.675(0.025)$ & $1.314(0.026)$ & $1.946(0.081)$ \\
HTC Desire X & Android & No & $1.243(0.054)$ & $1.817(0.075)$ & $1.462(0.087)$ \\
Galaxy Ace 2 & Android & No & $1.953(0.063)$ & $2.162(0.059)$ & $1.107(0.047)$ \\
Galaxy S2 & Android & No & $1.916(0.085)$ & $2.101(0.031)$ & $1.097(0.051)$ \\
Nexus One & Android & Yes & $1.514(0.051)$ & $1.568(0.045)$ & $1.036(0.046)$ \\
HTC One X & Android & Yes & $1.933(0.062)$ & $1.732(0.052)$ & $0.896(0.040)$ \\
HTC Wildfire S & Android & No & $2.032(0.088)$ & $1.683(0.063)$ & $0.828(0.047)$ \\
\hline
\end{tabular}

Table 2: Comparison of most popular smartphone devices. Values are means of ratios of 14 and $8 \mathrm{kHz}$ Goertzel filters, sampled every $\approx 3 \mathrm{~ms}(128$ samples at 44,100 kHz). Standard error of the mean (SEM) is given in brackets.

asked to select which insect their recording looks most similar. This promotes the involvement of the user in the process, who would otherwise be passively observing the detection performed on the smartphone.

\subsubsection{Possible Cicada Detected}

Figure $13 \mathrm{c}$ shows a message informing the user of the discovery of the cicada. Since the algorithm can be tricked by a recording of an actual call, the detection is presented as 'possible'.

\subsection{Evaluation of Microphones' Frequency Response}

Prior to deployment, it was noted that not all smartphones are equally capable of detecting the cicada. Our tests reveal that some smartphones are equipped with a microphone considerably more sensitive than others. We tested a range of different devices by reproducing four types of sound for at least 2 seconds each: silence, white noise, a frequency sweep from 50 to $20,000 \mathrm{~Hz}$, and the cicada call. These were reproduced in a custom-built sound-proof chamber, placed in a quiet location, with a Visaton KE 25 SC 8 Ohm tweeter producing the four test sounds. The phones were arranged with the microphone facing the speaker and all equally distant from it. From our experiences recording cicada calls in Slovenia, the sound volume was calibrated so that the volume of the cicada call was equivalent to that likely to be detected in the wild. The synthetic white noise and frequency sweep were tuned to match the maximum amplitude of the cicada call.

We report a comparison of the sensitivity of the microphones based on how well they detect the cicada call in our test environment. Table 2 summarises the outcome of the test, reporting the 


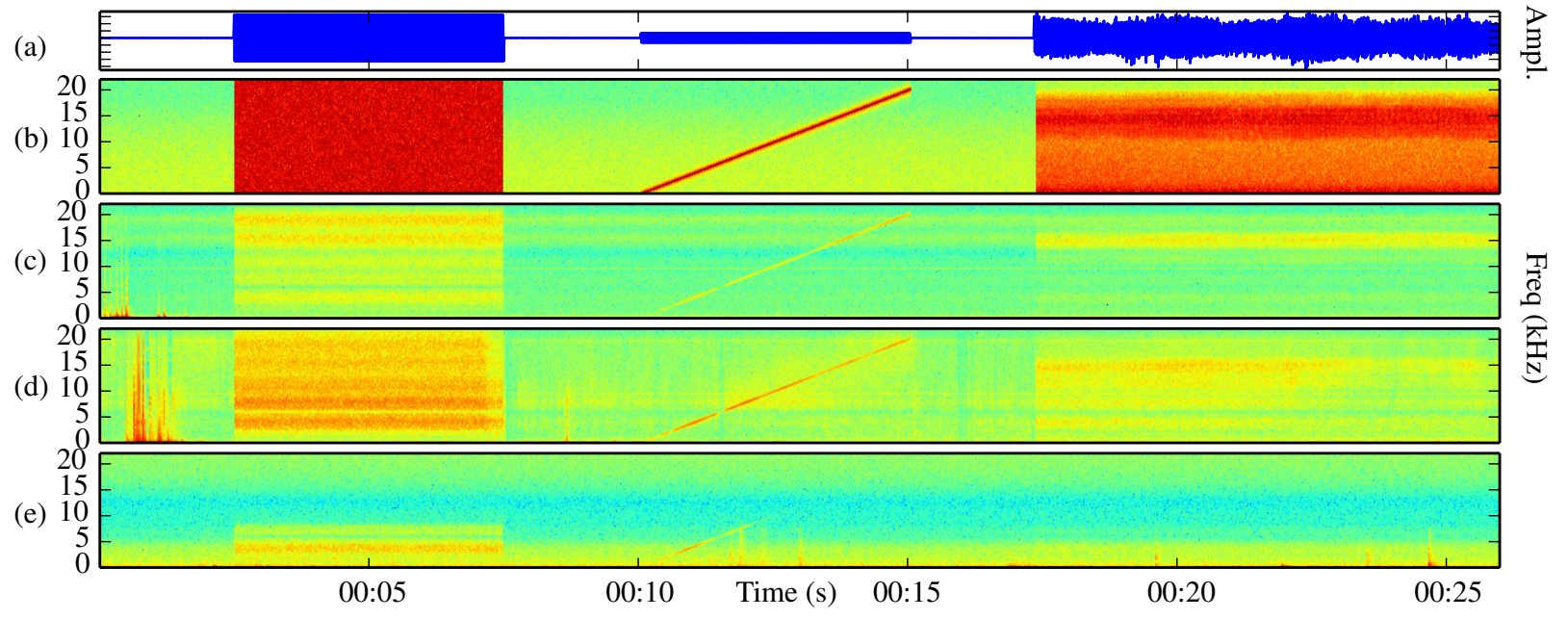

Figure 14: Comparison of three phones. At the top, the waveform (a) and spectrogram (b) of the sample calibration file. At the bottom, the very sensitive iPhone 5 (c), the Google Nexus 4 (d) and the hardware-filtered HTC One X (e), all top-end devices for iOS and Android.

ratio between the $14 \mathrm{kHz}$ and $8 \mathrm{kHz}$ bands extracted with the Goertzel filter when no sound was played (marked as Silence), when the cicada call was played (Cicada), and the ratio between these two. A higher value of the latter means a clearer indication of the cicada call, which results in a clearer separation of the log-normal distributions representing the sounded and unsounded states, and therefore greater confidence in the detection. It can be seen that all models of iPhone capture the call of the New Forest cicada most accurately, while Android phones exhibit a wide range in performance. This is not due to the operating system itself, but rather to the more varied range of hardware specifications in common Android devices. Figure 14 shows the reference sound played to the phone, together with three examples of high-end devices; the Apple iPhone 5, which detects the cicada call very clearly, the Nexus 4, which detects it most of the time, and the HTC One X, which despite having a sensitive microphone, uses a low-pass frequency filter, and is therefore incapable of detecting the insect's call. This is confirmed by the divergent rank these devices score in Table 2.

\subsection{Large-Scale Trial Deployment}

The smartphone app was launched on 8th June 2013 and collected data until the end of the mating season of the New Forest cicada. Since its launch, over 1000 citizen scientists have submitted over 6000 reports worldwide. Of these, at least 1777 were in the New Forest (over 1600 were submitted before a GPS fix had been acquired); of the New Forest reports, 162 were classified as either sounds interesting or potential cicada detected, and as a result include a 30 second audio recording. Of the citizen scientists who submitted reports, 738 used the iOS version of the app, while 346 used the Android version.

Figure 15 shows a bar graph of the number of reports uploaded by the top 25 contributors, with the trend for the top 100 users displayed in the top-right corner. It should be noted that among these, 5 are entomologists and authors of this paper. However, these users only covered specific areas of the forest, in particular those where the cicada had historically been observed. In contrast, the citizen scientists submitted much fewer reports per user, but the reports were much more evenly distributed 


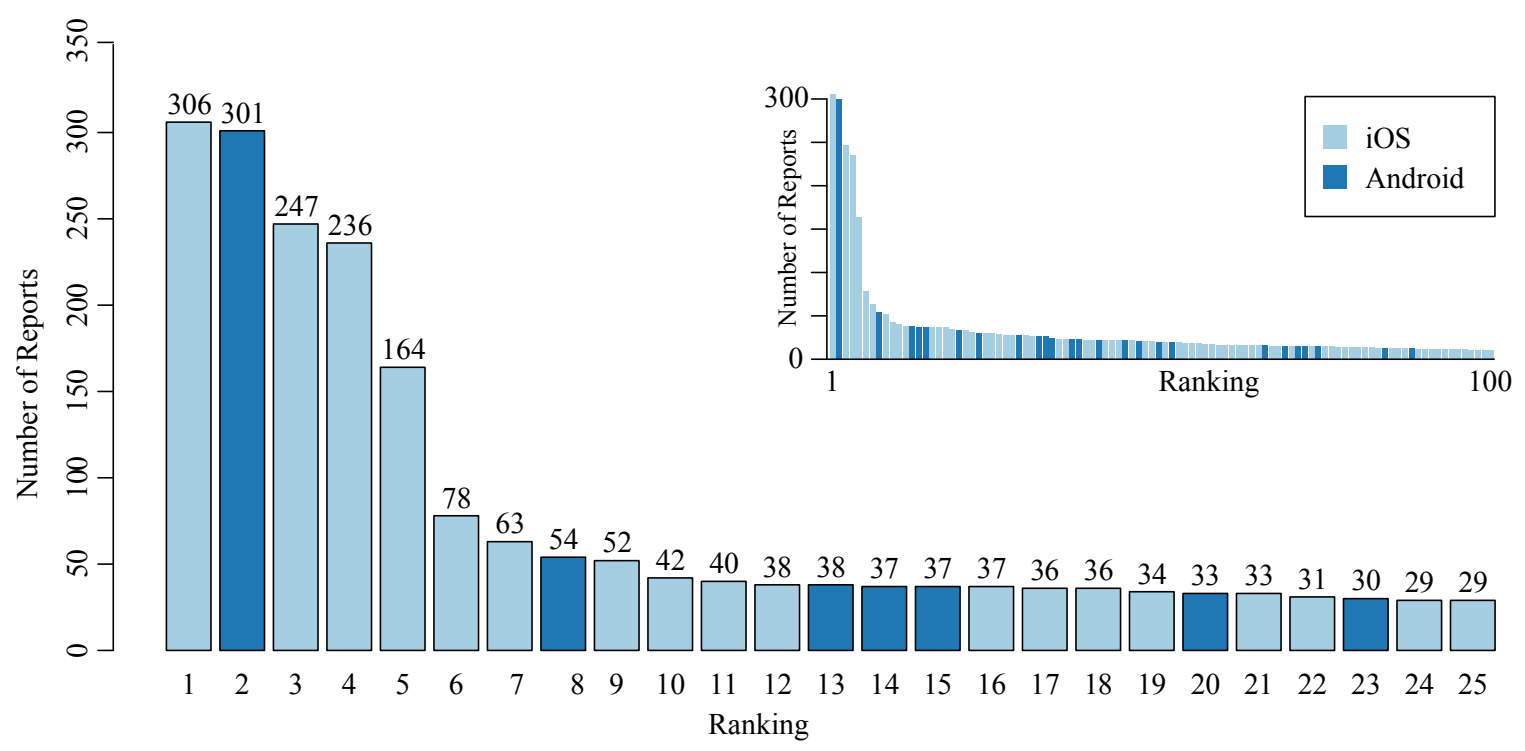

Figure 15: Reports per user by operating system for the top 25 users (right, trend of the top 100).

across the New Forest, as shown in Figure 16. This shows the crucial difference that this distributed approach can make, as entomologists cannot be ubiquitously present in different areas of the forest when the conditions are favourable, and can only cover a limited territory, while visitors, though contributing individually less, can help rediscover the cicada if it has moved to different sites, as it is currently suspected. At the same time, while entomologists have the tools and the knowledge to recognise insects' calls, the general public must be equipped with an accessible method. In this space, the implementation and deployment of our automated acoustic insect detection algorithm has succeeded to bring to the public the possibility to contribute to the distributed monitoring of insect species, as shown by the large number of downloads of the app and submitted reports.

\section{Conclusions}

In this paper we have presented a novel algorithm designed specifically to detect the mating call of the New Forest cicada. We have shown that through a careful analysis of its call, key features can be extracted at minimal cost, greatly simplifying the identification process. We compared our approach with three variants of our own approach, as no method exists to date to the best of our knowledge to automatically classify insects calls on a constrained platform, such as a mobile phone. Of the three variants, one uses the raw frequency components as HMM feature vectors, a second variant removes the silent periods of a recording and a third one classifies time slices independently based upon only the emission distributions. Our results show that our approach achieves an accuracy of $F_{1}=0.82$ for the detection of the New Forest cicada on a data set of recordings collected from the New Forest and Slovenia using iPhone and Android smartphones. Such recordings included various forms of background noise, insects' calls and human voices. Rather than focusing on the batch processing of large data sets of species, our approach is focused upon the identification of a small number of species in real time. 


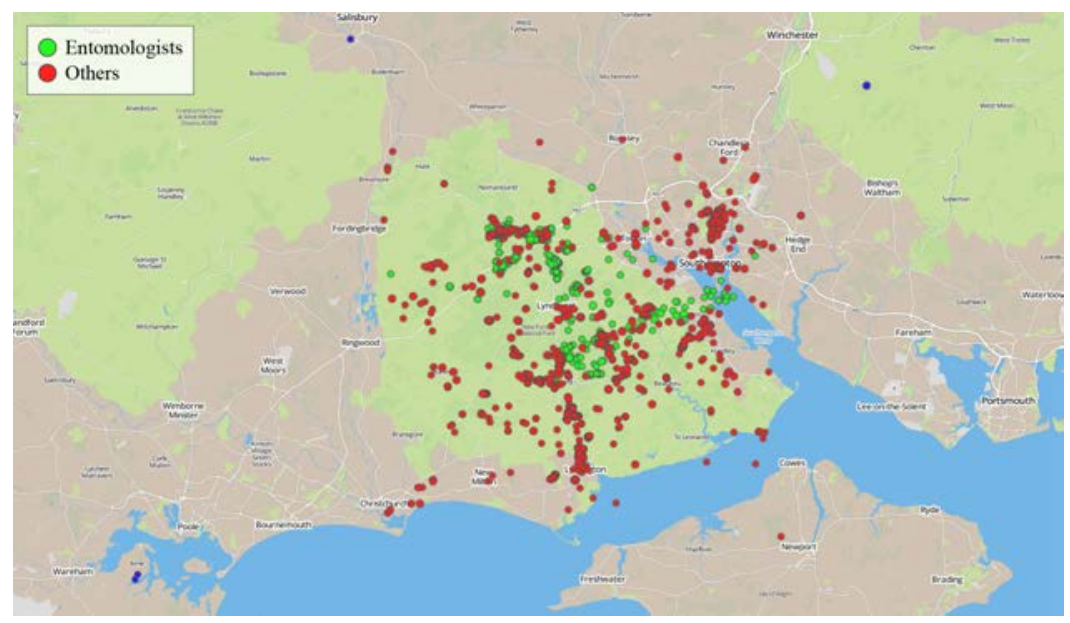

(a) The New Forest

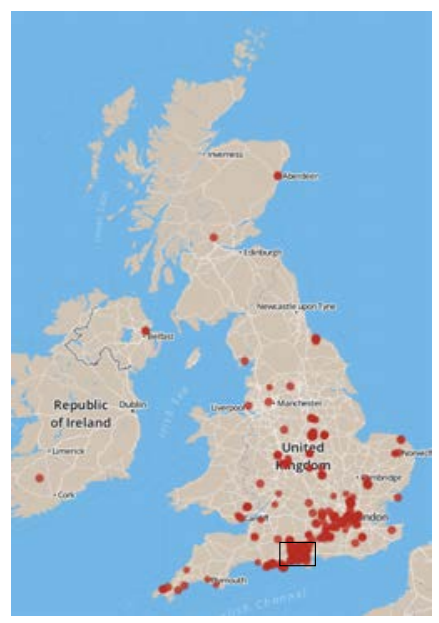

(b) UK

Figure 16: Map of submitted surveys around the New Forest and across the UK. The area of the New Forest corresponds to the green area in the centre.

With the development of the robust acoustic classifier complete, we integrated the technology into the smartphone app for iOS and Android. A large-scale deployment resulted in the collection of at least 1777 reports in the New Forest, of which 162 detected the call of an insect of interest, from over 1000 citizen scientists. Although the New Forest cicada was not successfully detected during its two month mating season in 2013, the use of the app in Slovenia confirmed the accuracy of the acoustic detector and the deployment in the New Forest attested the suitability of using citizen scientists to crowdsource the collection of audio reports via smartphones. The app is now also used by expert entomologists in Slovenia to detect the presence of Cicadetta montana.

Our future work will consist of a second deployment of the smartphone app during the two month mating season of the New Forest cicada, with the aim of achieving a greater coverage of the New Forest, and as such it will require the mobilisation of a larger community of citizen scientists to cover the areas of the New Forest which have not yet been surveyed. Such a deployment will constitute the largest survey of the New Forest cicada's habitat to date, and therefore will provide unprecedented insight into the existence of this endangered species. Moreover, an app for the classification of all British Orthoptera is also currently under development, and will pose a new set of challenges. In fact, with a higher number of different calls, the selection of distinctive features for the HMM becomes more difficult, and may require sampling at a higher frequency, increasing the computational complexity of the approach. To increase our accuracy and encompass a wider number of devices, we will use techniques such as cepstral mean normalisation to account for the difference in sensitivity of microphones.

Since the learning part of the algorithm is completed offline, our algorithm remains an efficient solution to classify insects' calls in real time on a mobile device, and may readily be extended to the calls of different animals, such as other insects and birds. Preliminary work on this extension has now started, with the goal of an adaptive acoustic classifier that can be trained for different sound-emitting wildlife species. 


\section{Acknowledgements}

This research was supported by an EPSRC Doctoral Training Centre grant (EP/G03690X/1) and by the ORCHID Project, www.orchid.ac.uk (EP/I011587/1). Many thanks to Dr Tomi Trilar and Prof Matija Gogala for their field guidance in Slovenia and to Dr David Chesmore for his original suggestion and ongoing support.

\section{References}

Brenna, B. (2011). Clergymen Abiding in the Fields: The Making of the Naturalist Observer in Eighteenth-Century Norwegian Natural History. Science in Context, 24(02), 143-166.

Chaves, V. A. E., Travieso, C. M., Camacho, A., \& Alonso, J. B. (2012). Katydids acoustic classification on verification approach based on MFCC and HMM. Proceedings of the 16th IEEE International Conference on Intelligent Engineering Systems (INES), 561-566.

Chesmore, E. D. (2004). Automated bioacoustic identification of species. Anais da Academia Brasileira de Ciências, 76(2), 436-440.

Chesmore, E. D., \& Ohya, E. (2004). Automated identification of field-recorded songs of four British grasshoppers using bioacoustic signal recognition. Bulletin of Entomological Research, 94(04), 319-330.

Dickinson, J. L., Zuckerberg, B., \& Bonter, D. N. (2010). Citizen Science as an Ecological Research Tool: Challenges and Benefits. Annual Review of Ecology, Evolution, and Systematics, 41(1), 149-172.

Ghahramani, Z. (2001). An Introduction to Hidden Markov models and Bayesian Networks. In Journal of Pattern Recognition and Artificial Intelligence, Vol. 15, pp. 9-42.

Go-Gulf (2012). Smartphone Users Around the World - Statistics and Facts. On-line, http://www.go-gulf.com/blog/smartphone, retrieved 19/07/2012.

Goertzel, G. (1958). An algorithm for the evaluation of finite trigonometric series. The American Mathematical Monthly, 65(1), 34-35.

Gomes, C. P. (2009). Computational Sustainability: Computational methods for a sustainable environment, economy, and society. The Bridge, 39(4), 5-13.

Joint Nature Conservation Committee (2010). UK priority species pages Cicadetta montana (New Forest Cicada). Tech. rep..

Jones, K. E., Russ, J., Catto, C., Walters, C., Szodoray-Paradi, A., Szodoray-Paradi, F., Pandourski, E., Pandourski, I., \& Pandourski, T. (2009). Monitoring bat biodiversity: indicators of sustainable development in Eastern Europe Darwin Initiative - Final Report. Tech. rep., Zoological Society London.

Josefsson, S. (2006). The base16, base32, and base64 data encodings. RFC 4648, Standards Track.

Leqing, Z., \& Zhen, Z. (2010). Insect Sound Recognition Based on SBC and HMM. In International Conference on Intelligent Computation Technology and Automation (ICICTA), Changsha, China, Vol. 2, pp. $544-548$.

MacLeod, N. (2007). Automated Taxon Identification in Systematics: Theory, Approaches and Applications. CRC Press. 
Miller-Rushing, A., Primack, R., \& Bonney, R. (2012). The history of public participation in ecological research. Frontiers in Ecology and the Environment, 10(6), 285-290.

Nature Locator (2012). BatMobile Project. On-line, http://batmobile.blogs.ilrt.org, retrieved $10 / 04 / 2014$.

Nature Locator (2013). iRecord Ladybirds Project. On-line, http://naturelocator.org/ladybird.html, retrieved 10/04/2014.

Pinchen, B. J., \& Ward, L. K. (2002). The history, ecology and conservation of the New Forest Cicada. British Wildlife, 13(4), 258-266.

Pinhas, J., Soroker, V., Hetzoni, A., Mizrach, A., Teicher, M., \& Goldberger, J. (2008). Automatic acoustic detection of the red palm weevil. Computer and Electronics in Agriculture, 63, 131139.

Potamitis, I., Ganchev, T., \& Fakotakis, N. (2006). Automatic acoustic identification of insects inspired by the speaker recognition paradigm.. In Interspeech 2006, Pittsburgh, Pennsylvania, pp. 2126-2129.

Quinn, J. A., Frias-Martinez, V., \& Subramanian, L. (2014). Computational Sustainability and Artificial Intelligence in the Developing World. AI Magazine Special Issue on Computational Sustainability.

Silvertown, J. (2009). A new dawn for citizen science.. Trends in ecology \& evolution, 24(9), $467-71$.

Viterbi, A. (1967). Error bounds for convolutional codes and an asymptotically optimum decoding algorithm. IEEE Transactions on Information Theory, 13(2), 260-269.

Zilli, D., Parson, O., Merrett, G. V., \& Rogers, A. (2013). A Hidden Markov Model-Based Acoustic Cicada Detector for Crowdsourced Smartphone Biodiversity Monitoring. In International Joint Conference for Artificial Intelligence, Beijing, China. AAAI Press. 